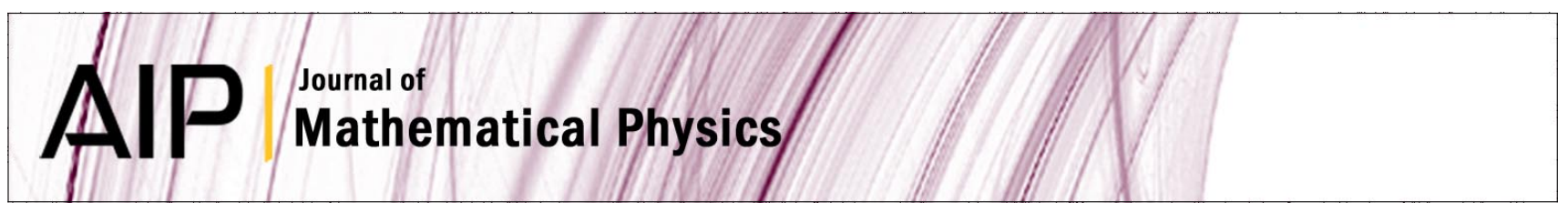

\title{
An inverse problem for a heat equation with piecewise-constant thermal conductivity
}

N. S. Hoang and A. G. Ramm

Citation: J. Math. Phys. 50, 063512 (2009); doi: 10.1063/1.3155788

View online: http://dx.doi.org/10.1063/1.3155788

View Table of Contents: http://jmp.aip.org/resource/1/JMAPAQ/v50/i6

Published by the American Institute of Physics.

\section{Related Articles}

Location of the Lee-Yang zeros and absence of phase transitions in some Ising spin systems J. Math. Phys. 53, 095211 (2012)

Sensitivity of the thermal and acoustic virial coefficients of argon to the argon interaction potential J. Chem. Phys. 137, 064702 (2012)

Entropy production in non-equilibrium fluctuating hydrodynamics

J. Chem. Phys. 137, 014509 (2012)

Burgers' equation and the sticky particles model

J. Math. Phys. 53, 063709 (2012)

On the accurate direct computation of the isothermal compressibility for normal quantum simple fluids:

Application to quantum hard spheres

J. Chem. Phys. 136, 244504 (2012)

\section{Additional information on J. Math. Phys.}

Journal Homepage: http://jmp.aip.org/

Journal Information: http://jmp.aip.org/about/about_the_journal

Top downloads: http://jmp.aip.org/features/most_downloaded

Information for Authors: http://jmp.aip.org/authors

\section{ADVERTISEMENT}

The most comprehensive support for physics in any mathematical software package World-leading tools for performing calculations in theoretical physics

Your work in Maple matches how you would write the problems and solutions by hand

State-of-the-art environment for algebraic computations in physics

The only system with the ability to handle a wide range of physics computations as well as pencil-and-paper style input and textbook-quality display of results

- Access to Maple's full mathematical power, programming language, visualization routines, and document creation tools 


\title{
An inverse problem for a heat equation with piecewise- constant thermal conductivity
}

\author{
N. S. Hoang ${ }^{\text {a) }}$ and A. G. Ramm ${ }^{\text {b) }}$ \\ Department of Mathematics, Kansas State University, Manhattan, Kansas 66506-2602, \\ USA
}

(Received 3 May 2009; accepted 28 May 2009; published online 24 June 2009)

The governing equation is $u_{t}=\left(a(x) u_{x}\right)_{x}, 0 \leq x \leq 1, t>0, u(x, 0)=0, u(0, t)=0$, $a(1) u^{\prime}(1, t)=f(t)$. The extra data are $u(1, t)=g(t)$. It is assumed that $a(x)$ is a piecewise-constant function and $f \not \equiv 0$. It is proved that the function $a(x)$ is uniquely defined by the above data. No restrictions on the number of discontinuity points of $a(x)$ and on their locations are made. The number of discontinuity points is finite, but this number can be arbitrarily large. If $a(x) \in C^{2}[0,1]$, then a uniqueness theorem has been established earlier for multidimensional problem, $x \in \mathbb{R}^{n}, n>1$ [see A. G. Ramm, Multidimensional inverse problems and completeness of the products of solutions to PDE, J. Math. Anal. Appl., 134, 211 (1988)] for the stationary problem with infinitely many boundary data. The novel point in this work is the treatment of the discontinuous piecewise-constant function $a(x)$ and the proof of Property $\mathrm{C}$ for a pair of the operators $\left\{\ell_{1}, \ell_{2}\right\}$, where $\ell_{j}:=-\left(d^{2} / d x^{2}\right)+k^{2} q_{j}^{2}(x), j$ $=1,2$, and $q_{j}^{2}(x)>0$ are piecewise-constant functions, and for the pair $\left\{L_{1}, L_{2}\right\}$, where $L_{j} u:=-\left[a_{j}(x) u^{\prime}(x)\right]^{\prime}+\lambda u, j=1,2$, and $a_{j}(x)>0$ are piecewise-constant functions. Property $\mathrm{C}$ stands for completeness of the set of products of solutions of homogeneous differential equations [see A. G. Ramm, Inverse Problems (Springer, New York, 2005)]. (C) 2009 American Institute of Physics.

[DOI: $10.1063 / 1.3155788$ ]

\section{INTRODUCTION}

Let

$$
\begin{gathered}
\dot{u}=\left(a(x) u^{\prime}\right)^{\prime}, \quad 0 \leq x \leq 1, \quad t>0, \quad u^{\prime}:=\frac{\partial u}{\partial x}, \quad \dot{u}:=\frac{\partial u}{\partial t}, \\
u(x, 0)=0, \quad u(0, t)=0, \quad a(1) u^{\prime}(1, t)=f(t) \not=0, \\
u(1, t)=g(t) .
\end{gathered}
$$

Problems (1) and (2) describe the heat transfer in a rod, $a(x)$ is the heat conductivity, $a(1) u^{\prime}(1, t)$ is the heat flux, $g(t)$ is the measurement, the extra data.

The inverse problem (IP) is as follows.

IP: Given $f(t)$ and $g(t)$ for all $t>0$, find $a(x)$.

Assumption A: $a(x)$ is a piecewise-constant function, $a(x)=a_{j}, x_{j} \leq x<x_{j+1}, x_{1}=0, x_{n+1}=1$, $0<c_{0} \leq a_{j} \leq c_{1}, 1 \leq j \leq n$.

This assumption holds throughout the paper and is not repeated. The set of piecewise-constant functions with finitely many discontinuity points is denoted by $\Pi$.

\footnotetext{
${ }^{a)}$ Electronic mail: nguyenhs@math.ksu.edu.

b) Author to whom correspondence should be addressed. Electronic mail: ramm@math.ksu.edu. 
If $a(x) \in C^{2}$, then the uniqueness of the solution to some multidimensional IPs has been proved in Ref. 6 (see also Ref. 5). Problems (1)-(3) with $a(x) \in C^{2}([0,1])$ has been studied in Refs. 8 and 9. The treatment of discontinuous piecewise constant $a(x)$ is of interest in applications. A different inverse problem for the heat equation was studied in 10.

In Ref. 4, Eq. (1) with the conditions $u(0, t)=u(1, t)=0, u(x, 0)=g(x)$, was studied, and the measured (extra) data were the values $u\left(\xi_{m}, t\right), \forall t>0,1 \leq m \leq M, 0 \leq \xi_{m} \leq 1$, where $M=3 n$ and $n$ is the number of the discontinuity points of $a(x)$. It was assumed in Ref. 4 that $\min _{j}\left|x_{j}-x_{j+1}\right|$ is not too small. Under these assumptions, the uniqueness theorem for the IP was proved in Ref. 4 and an algorithm for finding $a(x)$ was proposed. The stability of this algorithm with respect to perturbations of the data was not studied in Ref. 4 .

There is a large literature on IPs for the heat equation. We mention Refs. 1-3 and references therein.

In our paper, the extra data (3) consist of measurement, taken at one point, rather than at $3 n$ points, and we impose no restrictions on $\min _{j}\left|x_{j}-x_{j+1}\right|$. Under these assumptions, which are much weaker than in Ref. 4, we prove the uniqueness of the solution to IP.

One of our main results is as follows.

Theorem 1: The IP has at most one solution.

Remark 1: The IP is ill-posed: small variations in the data $\{f(t), g(t)\}$ in the $C(0, \infty)$-norm may lead to large variations in the coefficient $a(x)$ or may lead to a problem that has no solution. We assumed that the data are known for all $t>0$. If one assumes that $f(t)=0$ for $t>T$, where $T$ $>0$ is an arbitrary fixed number, then the solution $u(x, t)$ is an analytic function of $t$ in the region $t>T$. Therefore the data $\{f(t), g(t)\}$, known in the interval $[0, T+\epsilon)$, where $\epsilon>0$ is an arbitrary small fixed number, determine uniquely the data for all $t>0$. Thus, if $f(t)=0$ for $t>T$, then the uniqueness theorem for the solution to IP remains valid if the data are known for $t \in[0, T+\epsilon)$.

Let us formulate IP in an equivalent form.

Take the Laplace transform of Eqs. (1)-(3), denote

$$
v(x, \lambda):=L u:=\int_{0}^{\infty} e^{-\lambda t} u(x, t) d t,
$$

and get

$$
\begin{gathered}
\lambda v-\left(a(x) v^{\prime}\right)^{\prime}=0, \quad 0 \leq x \leq 1, \quad v(0, \lambda)=0, \\
a(1) v^{\prime}(1, \lambda)=F(\lambda), \quad v(1, \lambda)=G(\lambda),
\end{gathered}
$$

where $F:=L f$ and $G:=L g$.

The IP can be reformulated as follows.

IP: Given $F(\lambda)$ and $G(\lambda)$ for all $\lambda>0$, find $a(x)$.

Let us transform Eqs. (4) and (5) to yet another equivalent form.

Let $a(x) v^{\prime}:=\psi$. Then (4) and (5) can be replaced by the following problem:

$$
\begin{gathered}
-\psi^{\prime \prime}+\lambda a^{-1}(x) \psi=0, \quad \psi(1, \lambda)=F(\lambda), \quad \psi^{\prime}(0, \lambda)=0, \\
\psi^{\prime}(1, \lambda)=\lambda G(\lambda) .
\end{gathered}
$$

The IP can be reformulated as follows.

IP: Given $G(\lambda)$ and $F(\lambda)$, find $a^{-1}(x):=q^{2}(x)$.

Let

$$
\ell \psi:=-\psi^{\prime \prime}+k^{2} q^{2}(x) \psi=0, \quad \lambda:=k^{2}, \quad q^{2}(x):=a^{-1}(x), \quad c_{1}^{-1} \leq q^{2}(x) \leq c_{0}^{-1} .
$$

Consider the following problems: 


$$
\ell_{j} \psi_{j}=0, \quad \ell_{j}:=-\frac{d^{2}}{d x^{2}}+k^{2} q_{j}^{2}(x), \quad \psi_{j}^{\prime}(0, k)=0, \quad \psi_{j}(0, k)=1, \quad j=1,2 .
$$

Our second main result is as follows.

Theorem 2: The sets $\left\{\psi_{1}(x, k) \psi_{2}(x, k)\right\}_{\forall k \geq 0}$ and $\left\{v_{1}^{\prime}(x, \lambda) v_{2}^{\prime}(x, \lambda)\right\}_{\forall \lambda \geq 0}, k:=\lambda^{1 / 2}$, are dense in the set $\Pi$ of piecewise-constant functions on $[0,1]$.

Remark 2: Theorem 2 says that if $h(x) \in \Pi$ and

$$
\int_{0}^{1} h(x) \psi_{1}(x, k) \psi_{2}(x, k) d x=0, \quad \forall k>0,
$$

then $h=0$. Similar conclusion holds if $\psi_{j}(x, k)$ is replaced by $v_{j}^{\prime}(x, \lambda)$ in $(10)$. Such a property of the pair of the operators $\left\{\ell_{1}, \ell_{2}\right\}$ is called Property $C^{5,7}$

Clearly, if the set $\left\{\psi_{1}(x, k) \psi_{2}(x, k)\right\}_{\forall k \geq 0}$ is dense in the set $\Pi$, then the set of products $\left\{v_{1}^{\prime}(x, \lambda) v_{2}^{\prime}(x, \lambda)\right\}_{\forall \lambda \geq 0}$ is dense in the set $\Pi$.

In Sec. II proofs are given.

\section{PROOFS}

\section{A. Proof of Theorem 1}

Proof: We prove this theorem for problems (4) and (5). Suppose there are $v_{j}$ and $a_{j} \in \Pi, j$ $=1,2$, which solve problems (4) and (5), and let $w:=v_{1}-v_{2}$. Then

$$
\begin{gathered}
\lambda w-\left(a_{1} w^{\prime}\right)^{\prime}=\left(p v_{2}^{\prime}\right)^{\prime}, \quad p:=a_{1}(x)-a_{2}(x), \\
w(0, \lambda)=0, \quad w(1, \lambda)=0, \quad a_{1} v_{1}^{\prime}(1, \lambda)=a_{2} v_{2}^{\prime}(1, \lambda) .
\end{gathered}
$$

Multiply (11) by $v_{1}$, a solution to Eq. (4) with $a=a_{1}$, and integrate over $[0,1]$ and then by parts to get

$$
\int_{0}^{1} p(x) v_{2}^{\prime} v_{1}^{\prime} d x=\left.p v_{2}^{\prime} v_{1}\right|_{0} ^{1}+\left.a_{1} w^{\prime} v_{1}\right|_{0} ^{1}-\left.a_{1} w v_{1}^{\prime}\right|_{0} ^{1}=0, \quad \forall \lambda>0, \quad \lambda=k^{2}, \quad k>0,
$$

where we have used the conditions $w(0, \lambda)=w(1, \lambda)=0$ and $a_{1}(1) v_{1}^{\prime}(1, \lambda)=a_{2}(1) v_{2}^{\prime}(1, \lambda)$. Note that $v_{2}(x, \lambda)$ can be considered as an arbitrary solution to Eq. (4) up to a constant factor. The set $\left\{v_{1}^{\prime}(x, \lambda) v_{2}^{\prime}(x, \lambda)\right\}$ is dense in $\Pi$ by Theorem 2. Since $a_{1}(x)-a_{2}(x):=p(x) \in \Pi$, it follows from (13) that $p(x)=0$. So $a_{1}=a_{2}$. Theorem 1 is proved.

\section{B. Proof of Theorem 2}

Proof: Let us prove completeness of the set of products $\left\{\psi_{1}(x, k) \psi_{2}(x, k)\right\}_{\forall k \geq 0}$. Assume that $h \in \Pi$ and (10) holds. The function $\psi_{j}(x, k), j=1,2$, are entire functions of $k$. This follows from the integral equation for $\psi_{j}$, which is an immediate consequence of Eqs. (8) and (9),

$$
\psi_{j}(x, k)=1+k^{2} \int_{0}^{x}(x-s) q_{j}^{2}(s) \psi_{j}(s, k) d s, \quad x \geq 0, \quad j=1,2 .
$$

Equation (14) implies that for any fixed $k$, one has $\psi_{j}(x):=\psi_{j}(x, k) \geq 1, \forall x \in[0,1], j=1,2$, that $\psi_{j}^{\prime}(x, k) \geq 0, \quad \psi_{j}^{\prime \prime}(x, k) \geq 0$, and $\partial^{m} \psi_{j}(x, k) / \partial k^{m} \geq 0$ for all $m=0,1,2, \ldots$. Consequently, $\psi_{j}(x), j$ $=1,2$, are convex functions of $x$ on the semiaxis $x>0$. Since $\psi_{j}(x, k), j=1,2$, are positive, it follows from (14) that $\psi_{j}(x, k), j=1,2$, are increasing functions with respect to both $x$ and $k$. So we have

$$
\psi_{j}(x, k)>0, \quad \psi_{j}^{\prime}(x, k)>0, \quad \psi_{j}^{\prime \prime}(x, k)>0, \quad \forall k>0, \quad j=1,2 .
$$


Assume that $0<x_{11}<x_{12}<\cdots<x_{1 N_{1}}<1$ and $0<x_{21}<x_{22}<\cdots<x_{2 N_{2}}<1$ are discontinuity points of $a_{1}(x)$ and $a_{2}(x)$, respectively.

To derive from (10) that $h=0$, it is sufficient to prove that $h(x)=0, \forall x \in\left[x_{0}, 1\right]$, where $x_{0}$ $:=\max \left(x_{1 N_{1}}, x_{2 N_{2}}\right)$, because then one can prove similarly, in finitely many steps, that $h=0$ on the whole interval $[0,1]$ using the assumption $h \in \Pi$. We have

$$
\psi_{j}^{\prime \prime}(x, k)=k^{2} q_{j N_{j}}^{2}(x) \psi_{j}(x, k), \quad \forall k>0, \quad \forall x \in\left[x_{0}, 1\right],
$$

where $q_{j N_{j}}$ is the value of $q_{j}$ on the interval $\left[x_{0}, 1\right]$. From (16) one gets

$$
\psi_{j}(x, k)=a_{j}(k) e^{k q_{j N_{j}}\left(x-x_{0}\right)}+b_{j}(k) e^{-k q_{j N_{j}}\left(x-x_{0}\right)}, \quad \forall k \geq 0, \quad j=1,2 .
$$

It follows from (15) and (17) that

$$
\psi_{j}\left(x_{0}, k\right)=a_{j}(k)+b_{j}(k)>0, \quad \psi_{j}^{\prime}\left(x_{0}, k\right)=k q_{j N_{j}}\left[a_{j}(k)-b_{j}(k)\right] \geq 0
$$

and

$$
2 a_{j}(k)=\psi_{j}\left(x_{0}, k\right)+\frac{\psi_{j}^{\prime}\left(x_{0}, k\right)}{k q_{j N_{j}}}>\psi_{j}\left(x_{0}, k\right)
$$

This implies that

$$
a_{j}(k) \geq\left|b_{j}(k)\right| \geq 0, \quad \forall k>0, \quad j=1,2 .
$$

Since $h \in \Pi$, one may assume without loss of generality that

$$
h(x)=C \geq 0, \quad \forall x \in\left[x_{0}, 1\right] .
$$

It follows from (10) that

$$
\text { - } \int_{0}^{x_{0}} \psi_{1}(x, k) \psi_{2}(x, k) h(x) d x=\int_{x_{0}}^{1} \psi_{1}(x, k) \psi_{2}(x, k) h(x) d x, \quad \forall k>0 .
$$

From (15), (17), and (20), one gets

$$
1 \leq \psi_{j}(x, k) \leq \psi_{j}\left(x_{0}, k\right)<2 a_{j}(k), \quad 0 \leq x \leq x_{0}, \quad \forall k>0, \quad j=1,2 .
$$

Therefore,

$$
\left|\int_{0}^{x_{0}} \psi_{1}(x, k) \psi_{2}(x, k) h(x) d x\right| \leq 4 a_{1}(k) a_{2}(k) \int_{0}^{x_{0}}|h(x)| d x .
$$

From (15), (17), and (20) one obtains

$$
\psi_{j}(x, k) \geq a_{j}(k)\left[e^{k q_{j N_{j}}\left(x-x_{0}\right)}-e^{-k q_{j N_{j}}\left(x-x_{0}\right)}\right], \quad x \in\left[x_{0}, 1\right], \quad j=1,2 .
$$

Take an arbitrary $y \in\left(x_{0}, 1\right)$ and fix it. One has $\psi_{j}(x, k) \geq \psi_{j}(y, k), \forall x \in[y, 1]$. Therefore,

$$
\int_{x_{0}}^{1} \psi_{1}(x, k) \psi_{2}(x, k) h(x) d x \geq C(1-y) \psi_{1}(y, k) \psi_{2}(y, k), \quad \forall k>0 .
$$

Thus (22)-(24) imply the following inequalities:

$$
\infty>4 \int_{0}^{x_{0}}|h(x)| d x \geq C(1-y) \frac{\psi_{1}(y, k) \psi_{2}(y, k)}{a_{1}(k) a_{2}(k)}, \quad \forall k>0 .
$$

It follows from (25) that 


$$
\lim _{k \rightarrow \infty} \frac{\psi_{j}(y, k)}{a_{j}(k)}=\infty .
$$

Let $k \rightarrow \infty$ in (27) and use (28) to conclude that $C=0$ and, therefore, $h(x)=0$ for $x \in\left[x_{0}, 1\right]$. Similarly, one proves that $h(x)=0$ for all $x \in[0,1]$.

Theorem 2 is proved.

${ }^{1}$ Banks, H. and Kojima, F., "Boundary shape identification problems arising in thermal tomography," Inverse Probl. 6, 897 (1990)

${ }^{2}$ Bryan, K. and Caudill, L., "An inverse problem in thermal testing of materials," SIAM J. Appl. Math. 56, 715 (1996).

${ }^{3}$ Cannon, J., The One-Dimensional Heat Equation, Encyclopedia of Mathematics and Applications Vol. 23 (Cambridge University Press, Cambridge, 1984).

${ }^{4}$ Gutman, S. and Ha, J., "Identifiability of piecewise constant conductivity in a heat conduction process," SIAM J. Control Optim. 46, 694 (2007).

${ }^{5}$ Ramm, A. G., Inverse Problems (Springer, New York, 2005).

${ }^{6}$ Ramm, A. G., Multidimensional Inverse Scattering Problems (Longman, New York/Wiley, New York, 1992).

${ }^{7}$ Ramm, A. G., "Property C for ODE and applications to inverse problems," Operator Theory and Its Applications (Amer. Math. Soc., Fields Institute Communications, Providence, RI, 2000), Vol. 25, pp. 15.

${ }^{8}$ Ramm, A. G., "An inverse problem for the heat equation," J. Math. Anal. Appl. 264, 691 (2001).

${ }^{9}$ Ramm, A. G., "An inverse problem for the heat equation II," Appl. Anal. 81, 929 (2002).

${ }^{10}$ Ramm, A. G., "Inverse problems for parabolic equations," Austral. J. Math. Anal. Appl. (AJMAA) 2, 1 (2005). 